\title{
Ferrofluid Thin Films for Airfoil Lift Enhancement and Delaying Flow Separation
}

\author{
Francisco J. Arias ${ }^{1} \dagger$ \\ ${ }^{1}$ Department of Fluid Mechanics, Polytechnic University of Catalonia, \\ ESEIAAT C/ Colom 11, 08222 Barcelona, Spain
}

(Received $\mathrm{xx}$; revised $\mathrm{xx}$; accepted $\mathrm{xx}$ )

In this work, consideration is given to a novel concept for airfoil lift enhancement and delaying flow separation. Here, lift enhancement is attained by preventing the growth of the boundary layer from the elimination of the zero slip condition between the wing surface and the air stream. The concept would simulate all the effects of a moving wall leading in the appearance of slip velocity in the gas-fluid interface including the injection of momentum into the air boundary layer, but with one exception: here there is no moving wall but instead a ferrofluid thin film pumped parallel and attached at the wall by a magnetic field. Utilizing a simplified physical model for the profile velocity of the ferrofluid film and from ferrohydrodynamic stability considerations an analytical expression for the interfacial velocity was derived. Finally, from the available experimental data on moving walls the expected lift and attack angle enhancement was found and the weight-penalty per unit of surface area of the wing estimated. Additional R\&D is required in order to explore the possibilities in the use of ferrofluid thin films.

\section{Nomenclature}

$g=$ gravity

$H=$ magnetic field

$l=$ thickness of the permanent magnet or ferrofluid

$M=$ magnetization

$p=$ pressure

$Q=$ volumetric flow

$S=$ surface area of the wing

$T=$ temperature

$u=$ velocity of air

$v=$ velocity of ferrofluid

$W=$ weight

$x=$ length coordinate

$z=$ normal coordinate

\section{Greek symbols}

$\delta=$ thickness of the ferrofluid film

$\eta=$ dynamic viscosity

$\mu=$ magnetic momentum

$\rho=$ density

$\dagger$ Email address for correspondence: francisco.javier.arias@upc.edu 
$\sigma=$ surface tension

\section{subscripts}

$a=$ air

$f=$ ferrofluid

$i=$ interface air-ferrofluid

$m=$ magnet

$o=$ interface

\section{Introduction}

Almost immediately after the Prandtl's boundary layer theory was proposed, continuous research has been performed in order to find methods to mitigate its negative effects. Despite the multitude of approaches to tackle the problem, however, all of them in one way or another try to prevent, or at least delay, the detachment of the boundary layer from the wall, (e.g. Modi. 1997; Hammer et al. 2018; Fernandez et al. 2018; Panda et al. 1994; Bhat et al. 2019; Yeh et al. 2019; Gao et al. 2019; Wang et al. 2019; Ringuette et al. 2007; Gilarranz et al, 2005). Suction and blowing, turbulence promoters, vortex generator, and moving walls are just some examples of the several techniques which can be found in the literature. By Suction and blowing is intended to remove low energy air, either through suction slots or by blowing high-energy air through backward-directed slots, respectively. On the other hand, turbulence promoters and vortex generators attempt to control flow separations of symmetric airfoils by creating spots or high turbulence by using some elements such as baffles or wall roughness elements, and finally we have moving solid walls which are intended to remove the zero slip condition as well as injecting momentum into the boundary layer.

The object of this work was to analyze a novel approach for lift enhancement. In this concept, the goal is attained by preventing the growth of the air boundary layer from the elimination of the zero slip condition between the surface and the air stream and more exactly by elimination of the air-wing direct contact. The concept would simulate all effects of a moving wall leading in the appearance of slip velocity in the gas-fluid interface including the injection of momentum into the boundary layer, with one exception: there is no moving wall but instead a ferrofluid thin film pumped parallel and attached at the wall by a magnetic field. For this work, suffice is to know that a ferrofluid or ferromagnetic fluid is nothing more than a colloidal liquid that becomes strongly magnetized in the presence of a magnetic field due to presence of suspended nanoscale ferromagnetic, or ferrimagnetic particles. For the sake of illustration, Fig. 1 shows a pictorially illustration of the concept investigated in this work, however, caution is called. This figure is not intended to give a definitive design. Nor should it be misconstrued as an attempt to produce a definitive optimized application of the concept, where, real applications could depart largely from this sketch, nonetheless, this will provide important guidance above the core idea proposed in this work. 


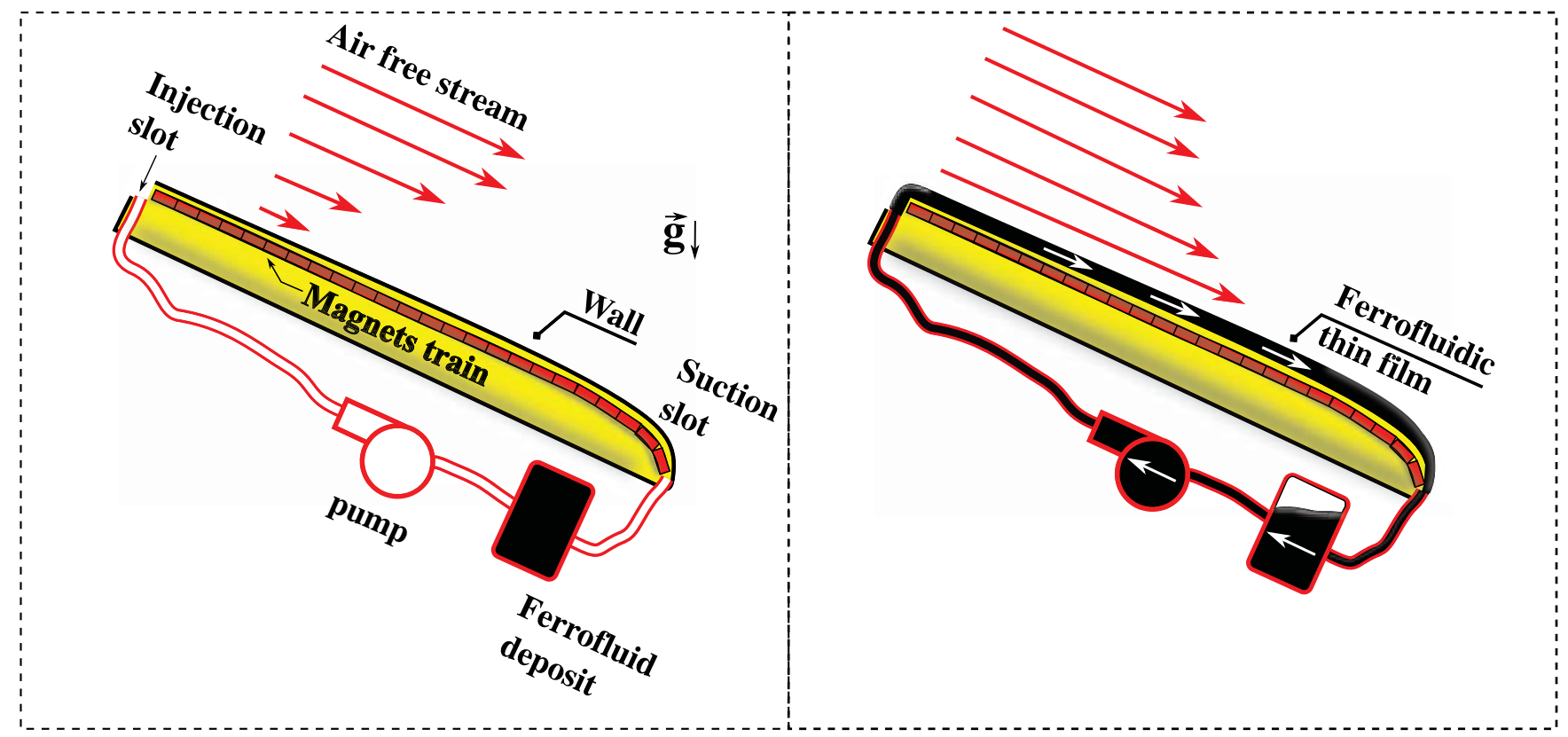

FiguRE 1. Sketch of the core idea. Left. In normal conditions, the zero slip condition leads to the formation of a boundary layer limiting the lift. Right. Because the injection of a ferrofluid thin film -attached at the wall by a magnetic field, the zero slip condition disappear which translates into lift enhancement.

\section{Materials and Methods}

\subsection{The ferromagnetic thin film layer}

To begin with, let us consider Fig. 2 in which a ferrofluid thin film ( with a few millimeters thickness or less) is attached at the wall of an airfoil by a magnetic gradient field normal to the surface which is generated by, say, an array of permanent magnets attached below the wing. In addition, the ferrofluid is pumped tangentially to the surface of the wall. We choose the normal co-ordinates to the surface as $z$-axis and the $x$-axis in the direction of motion of the fluid and we are fixing our origin of coordinates at the wall. Considering that the velocity depends only on the normal axis $z$, and that the film of which we are dealing is with a thickness of a few millimeters or less, then, the convective term in the Navier-Stokes can be neglected in comparison with viscous term, and then we have (Rosenweig 1985)

$$
\frac{1}{\eta_{f}} \frac{\partial p}{\partial x}=\frac{\partial^{2} v_{x}}{\partial z^{2}}
$$

and

$$
\frac{\partial p}{\partial y}=\rho_{f} g+\mu_{f} M_{f} \frac{\partial H}{\partial z}
$$

where $p$ is pressure; $v_{x}$ is the ferrofluid velocity parallel to the wall; $\eta_{f}, \rho_{f}, M_{f}$ and $\mu_{f}$ are the dynamic viscosity, density, magnetization and magnetic momentum of the ferrofluid, respectively; $g$ is gravity, and $\frac{\partial H}{\partial z}$ is the uniform normal magnetic gradient. After integrating Eq.(2.2) one obtains

$$
p=p_{1}(x)+\rho_{f} g z+\mu_{f} M_{f} \frac{\partial H}{\partial z} z
$$




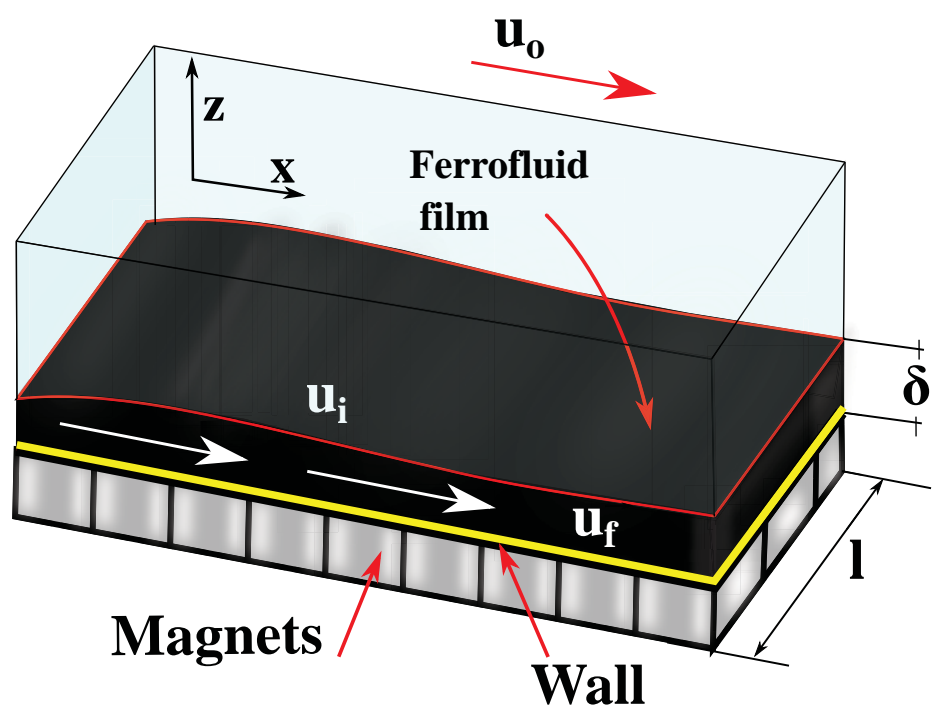

Figure 2. Physical model of the region of the airfoil covered by the ferrofluid film

Now, we will define the boundary conditions for Eq.(2.1). First, on the solid boundary the ferrofluid velocity vanishes, it give us the first boundary condition

$$
v_{x}(z=0)=0
$$

Second, on the interface air-ferrofluid, $z=\delta$ the components of a viscous-stress tensor are continuous, (Landau \& Lifshitz 1989), and then

$$
\left.\eta_{f} \frac{\partial v_{x}}{\partial z}\right|_{z=\delta}+\left.\eta_{a} \frac{\partial u_{x}}{\partial z}\right|_{z=\delta}=0
$$

where $u$ and $\eta_{a}$ are the air velocity and dynamic viscosity, respectively. For a very thin film $\delta \rightarrow 0$ and considering that $\eta_{f} \gg \eta_{a}$, it could be allowable to assume $\eta_{f} \frac{\partial v_{x}}{\partial z} \gg \eta_{a} \frac{\partial u_{x}}{\partial z}$ and then Eq.(2.5) simplify as

$$
\eta_{f} \frac{\partial v_{x}}{\partial z} \approx 0
$$

Finally, the discharge or volumetric flow is given by

$$
Q=l \int_{0}^{\delta} v_{x}(z) d z
$$

Taking into account the set of boundary conditions, the solution of Eq.(2.1) yields

$$
v_{x}=\frac{3 Q}{2 l \delta^{2}}\left[2 z-\frac{z^{2}}{\delta}\right]
$$

The above equation is familiar with that from a Couette flow with pressure gradient as was expected. (Rowell 1928) and (Bateman 1932). On the other hand, the interfacial velocity, $v_{x}(z=\delta)=v_{i}$

$$
v_{i}=\frac{3 Q}{2 l \delta}
$$




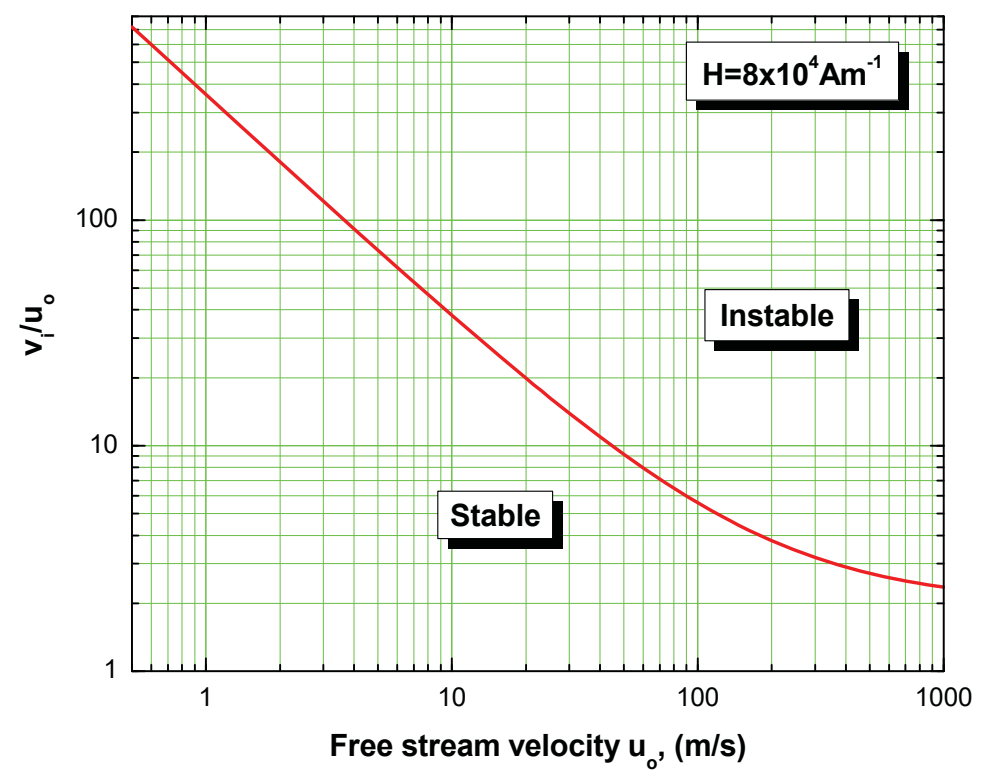

FIGURE 3. Stability curve predicted by Eq.(2.13) as a function of the air free stream

likewise the mean velocity $\bar{v}_{x}$

$$
\bar{v}_{x}=\frac{Q}{\delta l}
$$

which considering Eq.(2.9) becomes

$$
\bar{v}_{x}=\frac{2 v_{i}}{3}
$$

\subsection{Film stability}

From Eq.(2.8) one may be tempted to think that by increasing the volumetric flow indefinitely, i.e., the pumping power, or by decreasing the thickness of the film, it could be possible to increase the interface velocity as pleased. However, this is not the case. Actually, the maximum interface velocity is limited by Kelvin-Helmholtz instabilities which arose from the relative motion between the ferrofluid and the air stream. The criterion for instability in the magnetic Kelvin-Helmholtz problem when the ferrofluid film is under the action of a magnetic field is given by, (Rosenweig 1985)

$$
\left(\bar{v}_{x}-u_{o}\right)^{2}>\frac{\rho_{f}+\rho_{a}}{\rho_{f} \rho_{a}}\left[2\left[g\left(\rho_{f}-\rho_{a}\right) \sigma\right]^{\frac{1}{2}}+\frac{\left(\mu_{a}-\mu_{f}\right)^{2} H_{x}^{2}}{\mu_{a}+\mu_{f}}\right]
$$

where $\bar{v}_{x}$ is the mean ferrofluid velocity, $u_{o}$ the air free stream velocity; $\rho_{f}$ and $\rho_{a}$ the density of the ferrofluid and the air, respectively; $g$ is gravity; $\sigma$ the surface tension, $\mu_{a}$ and $\mu_{f}$ the magnetic permeability of the air and the ferrofluid, respectively. In the above equation, the uniform magnetic field $H_{x}$ is the magnetic field collinear with the direction of wave propagation (the stream direction), where it is known that a tangential applied magnetic field in the direction normal to the direction of wave propagation offers no stabilization, (Rosenweig 1985).

Because $\rho_{a} \ll \rho_{f}$ and $\mu_{a} \ll \mu_{f}$, and taking into account Eq.(2.11), Eq.(2.12) becomes 


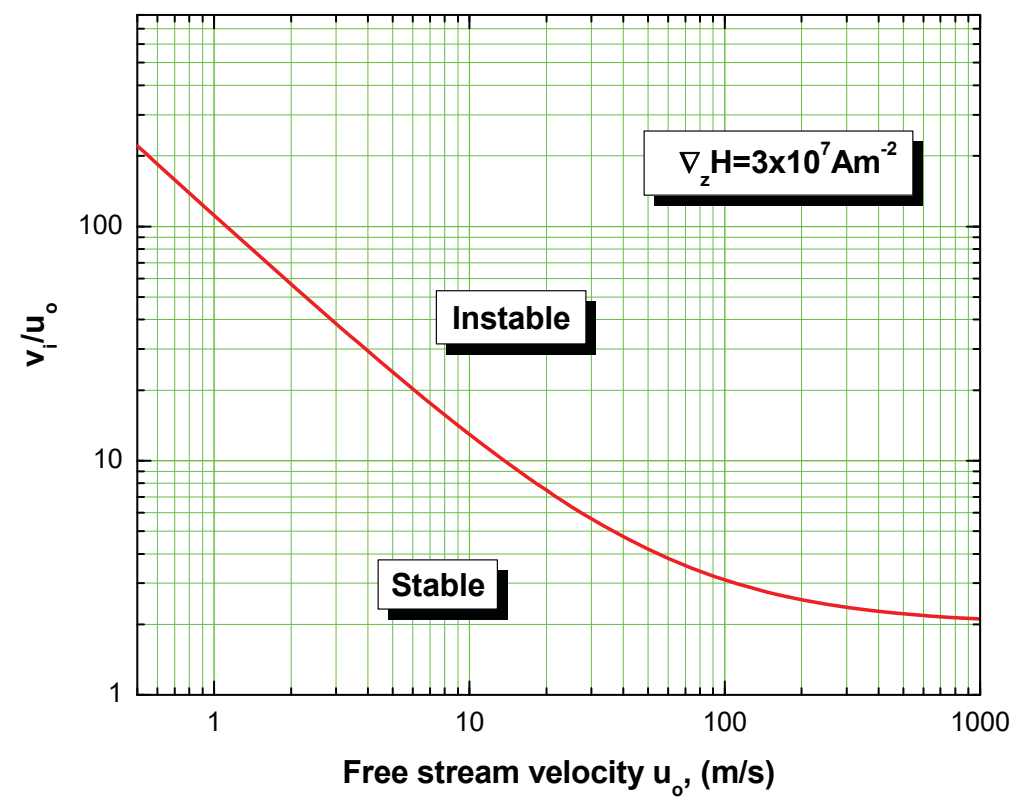

Figure 4. Stability curve predicted by Eq.(2.16) as a function of the air free stream

$$
\frac{v_{i}}{u_{o}}>\frac{3}{2}\left[1+\frac{1}{u_{o}}\left[\frac{2\left(g \rho_{f} \sigma\right)^{\frac{1}{2}}+\mu_{f} H_{x}^{2}}{\rho_{a}}\right]^{\frac{1}{2}}\right]
$$

However, despite that a uniform magnetic field normal to the direction of wave propagation provides null stabilization, nevertheless, a magnetic gradient in this direction is causing a normal body force per unit of volume as

$$
F_{m}=\mu_{o} M_{f} \nabla_{z} H
$$

where $\mu_{o}$ is the permeability of free space; $M_{f}$ the magnetization of the ferrofluid, and $\nabla_{z} H$ is the normal magnetic field gradient. Thus, because both gravity and the magnetic force are body forces, an effective acceleration $g_{e}$ may be defined as

$$
g_{e}=g+\frac{\mu_{o} M \nabla_{z} H}{\rho_{f}}
$$

Therefore, if only a gradient of magnetic field is acting on the ferrofluid plus gravity and surface tension, the criterion of stability, Eq.(2.13) becomes

\section{- Discussion}

$$
\frac{v_{i}}{u_{o}}>\frac{3}{2}\left[1+\frac{1}{u_{o}}\left[\frac{2 \sigma^{\frac{1}{2}}\left(g \rho_{f}+\mu_{o} M_{f} \nabla_{z} H\right)^{\frac{1}{2}}}{\rho_{a}}\right]^{\frac{1}{2}}\right]
$$

In order to obtain some idea of the shape of the curves predicted by Eq.(2.13) and Eq.(2.16), we assume some typical values of the parameters for a ferrofluid: $\sigma=70 \times 10^{-3}$ $\mathrm{N} / \mathrm{m} ; g=9.8 \mathrm{~m} / \mathrm{s}^{2} ; \rho_{f}=1.2 \times 10^{3} \mathrm{~kg} / \mathrm{m}^{3} ; \rho_{a}=1.0 \mathrm{~kg} / \mathrm{m}^{3} ; M_{f}=4.5 \times 10^{5} \mathrm{~A} / \mathrm{m}$ which corresponds to a realizable magnetic field around $0.5 \mathrm{~T}$ obtained from a typical hand-held permanent magnet; $\mu_{o}=4 \pi \times 10^{-7} \mathrm{H} /(\mathrm{m}) ; \mu_{f}=8 \mu_{o}$. The resulting curves are shown 


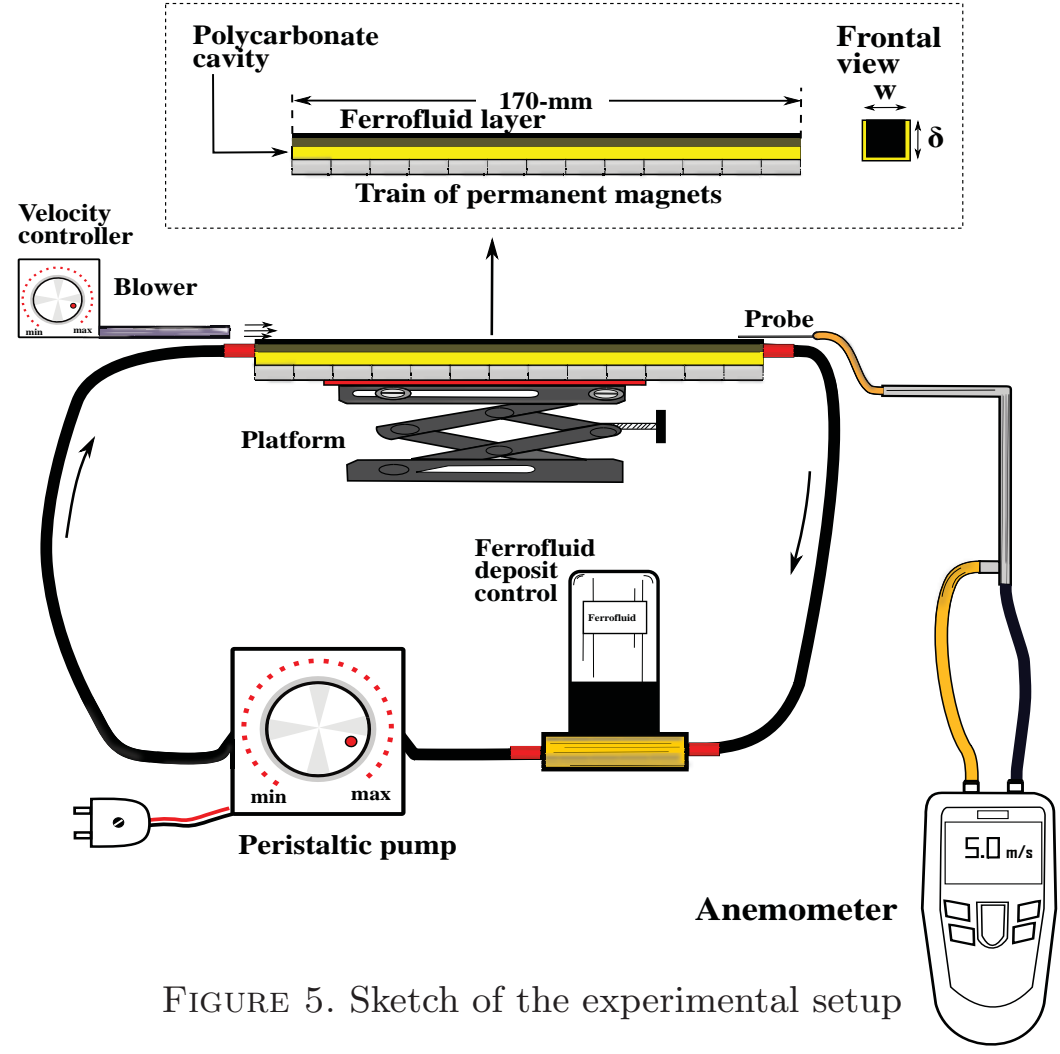

in Fig. 3 and Fig. 4 for Eq.(2.13) and Eq.(2.16), respectively, and considering practical achievable values for the magnetic field and the magnetic gradient as well.

\subsection{Experimental measurement}

In order to obtain some preliminary idea of the lift enhancement by the use of ferrofluid thin films full experiments on specific airfoil designs are not required. Instead owing that from an aerodynamic point of view there is not substantial difference if the interfacial velocity is generated by a thin film or, say, a moving solid surface, then the thin film can be regarded to some extent as a moving solid surface (both are introduced into the differential equation of Navier Stokes as a simple boundary condition) and thus it is possible to take advantage of the available experimental measurements in the literature on lift enhancement and flow separation from moving solid surfaces for several airfoil designs. Hence after obtaining experimental measurements of the interfacial velocity from the ferrofluid film,

the lift and angle of attack can be inferred from the available literature on moving surfaces with the same interfacial velocity. With this goal, a set of experiments were performed to find the attainable interfacial velocity by the notion of the ferrofluid film.

The setup consisted in a square polycarbonate cavity $\delta=l$ and 170-mm-long filled with ferrofluid. Below the cavity a train of hand-held neodymium permanent magnets were located. The ferrofluid was pumped through the cavity by using a peristaltic pump electronically regulated by the number of revolutions per minute. Several cavities were used, i.e, different $\delta$ but keeping always the same length. The ferrofluid employed was $\mathrm{Mn}_{0.5} \mathrm{Zn} 0.5 \mathrm{Fe}_{2} \mathrm{O}_{4}$ in water at room temperature $T=298 \mathrm{~K}$. The air stream was propelled by a blower parallel to the cavity. Interfacial velocity $v_{i}$ was measured by using a Fluke 922 airflow meter. The cavity was positioned at a simple laboratory adjustable liftingrotating platform which allows the measurement at different angles of inclination. The magnetic field from the array of magnets was measured as $0.12 \mathrm{~T}$ at $3 \mathrm{~mm}$ from its 


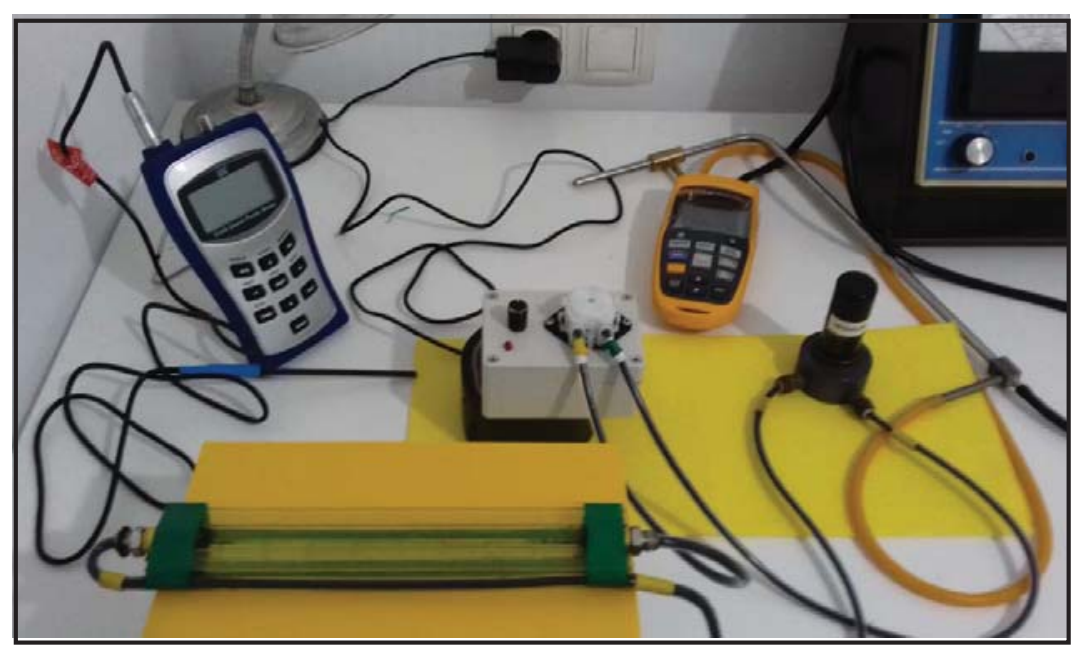

Figure 6. Closeup of experimental setup

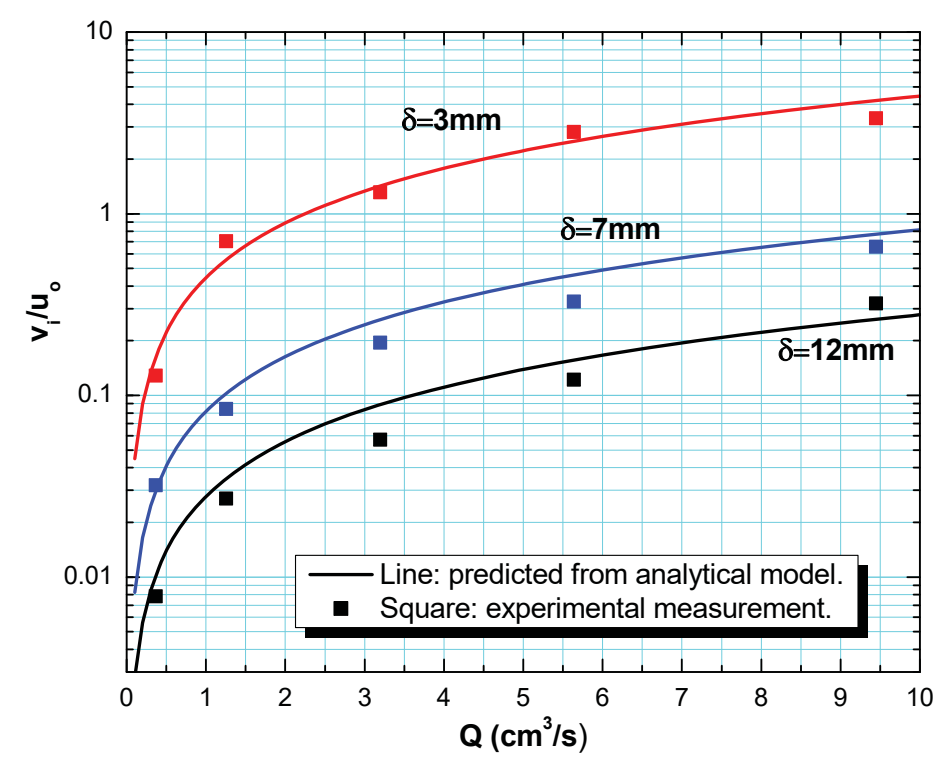

FiguRE 7. $\frac{v_{i}}{u_{o}}$ as a function of the volumetric flow with $u_{o}=0.5 \mathrm{~m} / \mathrm{s}$.

surface using a FW BELL 5170 Gauss/Tesla meter. Figs. 5 an 6 show the sketch and real experimental setup, respectively.

\section{Results and conclusions}

The resulting experimental curves are shown in Figs. 7, 8 and 9. Fig. 7 shows the ratio $\frac{v_{i}}{u_{o}}$ as function of the volumetric flow and for several thickness of the cavity. Fig. 8 shows the ratio $\frac{v_{i}}{u_{o}}$ as function of the free stream air; and Fig. 9 shows the effect of the angle of inclination (by taking measurements at different inclination angles of the platform from a totally horizontal inclination $0^{\circ}$ to a total vertical inclination $90^{\circ}$, see Fig. 5) of the cavity on the ratio $\frac{v_{i}}{u_{o}}$ and for several thickness of the cavity.

Finally, Fig. 10 and Fig. 11 show the predicted lift enhancement and the increase 


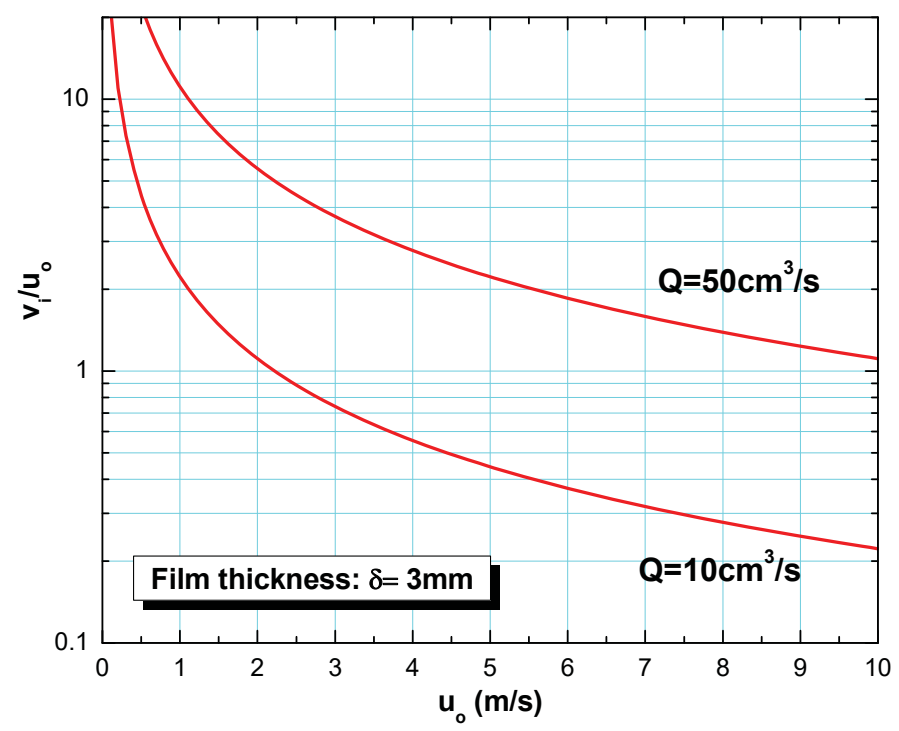

FIGURE 8. $\frac{v_{i}}{u_{o}}$ as a function of the free stream air

of angle of attack for a NACA0015 derived from using the experimental interfacial velocity obtained before and applied to the plots from experimental data on moving walls reported by Boukenkoul et al.2017., i.e, by replacing the velocity of the wall by the interfacial velocity of the film.

It is easy to see the attractiveness of the proposed concept by comparing the lift coefficient enhancement as function of $\frac{v_{i}}{u_{o}}$ from Fig. 10, and the allowable $\frac{v_{i}}{u_{o}}$ as function of the air stream velocity from the curves of ferrohydrodynamic stability Fig. 3 and Fig. 4. Thus, as an illustrative example, an air stream velocity around $100 \mathrm{~m} / \mathrm{s}$ will allow for a thin film $\frac{v_{i}}{u_{o}}$ ratio around $\approx 3 \mathrm{~m} / \mathrm{s}$ before Kelvin Helmholtz will detach the film. With this ratio, the lift coefficient enhancement could be around 1.8 which is a figure of merit to be considered. Additional $\mathrm{R} \& \mathrm{D}$ is required in order to explore the possibilities in the use if ferrofluid thin films.

\subsection{The weight-penalty}

There is not doubt that one of the major factors to be considered in an aircraft project is the weigh. In the application of the proposed method, one of the main concerns could be related with the weight of the magnets needed to attach the ferrofluid film. However, because the ferrofluid film is very thin -a few millimeters or less, it is expected that the extra weight from the train of magnets attached at the wing of the aircraft will not result in additional concerns. In order to assess the extra weight per surface area of the wing caused by the magnets we will proceed as follows: First, we need to determine the external magnetic field needed to attain the magnetic saturation of the ferrofluid. The measurement of the magnetization of the ferrofluid as function of the external magnetic field is shown in Fig. 12. In this figure it is easy to see that with an external magnetic field around $100 \mathrm{kA} / \mathrm{m}$ we attain the $10 \%$ of the saturation $(\approx 32 \mathrm{kA} / \mathrm{m})$ which translates into $\approx 3.2 \mathrm{kA} / \mathrm{m}$ which for a film with a few millimeters will result into gradients $>10^{5} \mathrm{~A} / \mathrm{m}^{2}$ which is enough to guarantee a strong film attachment at the wall (see Fig. 3).

Second, we need to asses, the amount of magnets needed to generate the magnetic 


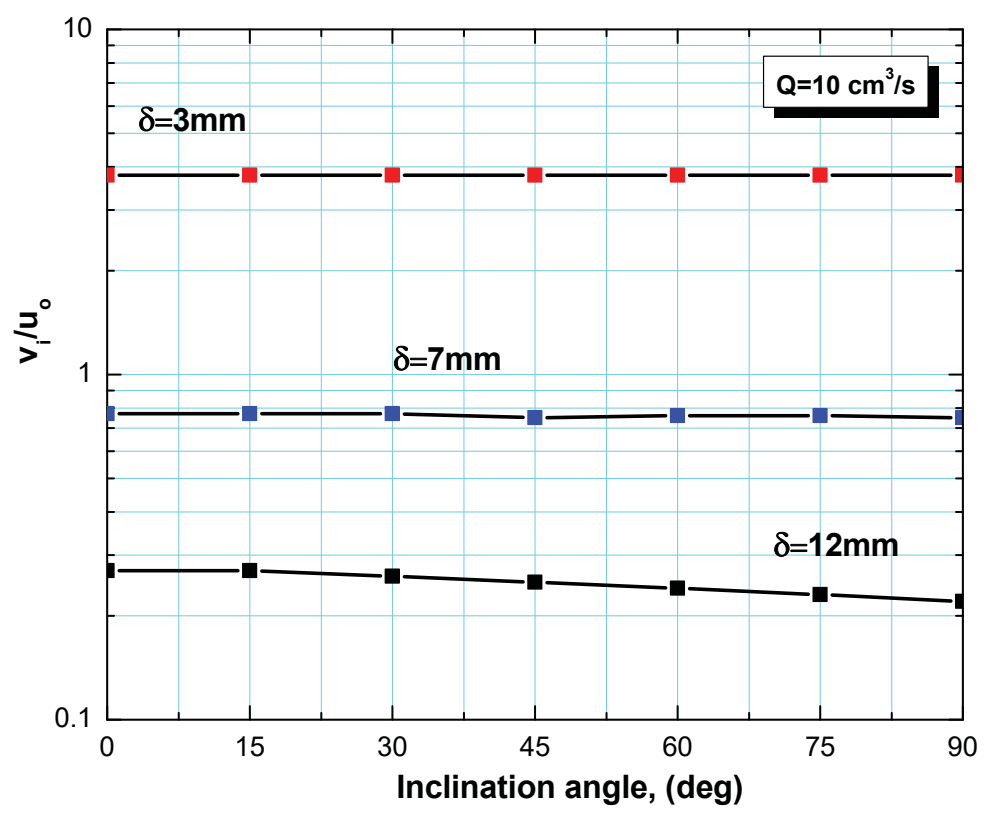

FIGURE 9. $\frac{v_{i}}{u_{o}}$ as a function of the angle of inclination and for several thickness of the cavity

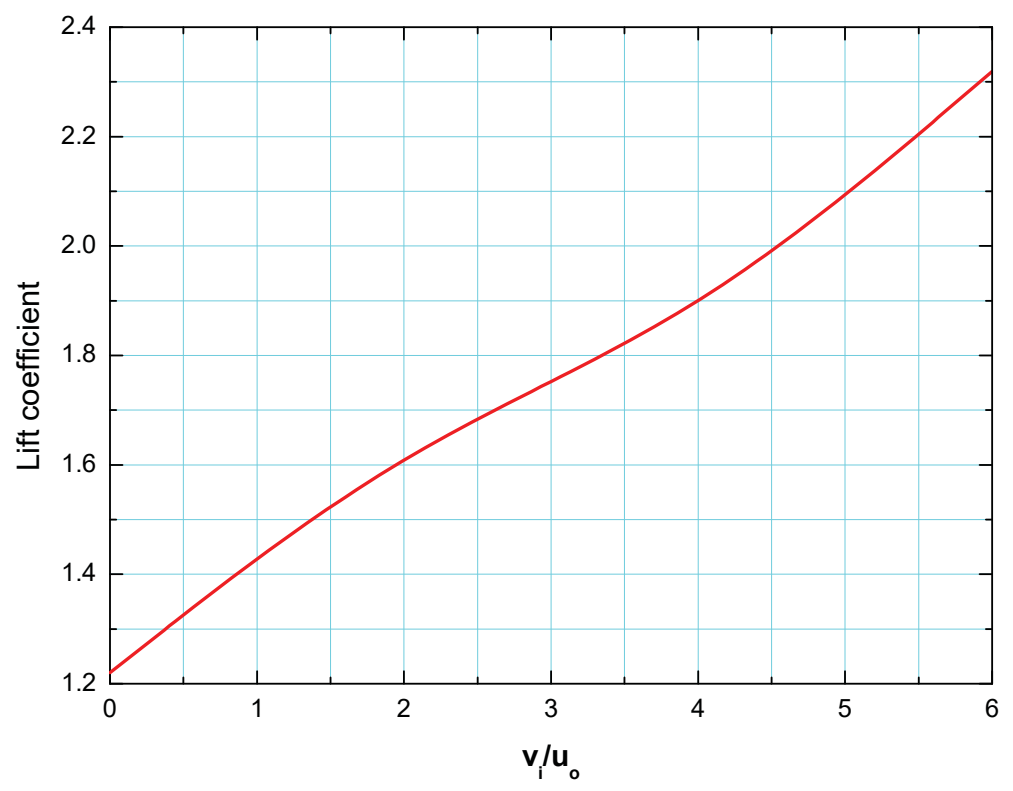

Figure 10. Lift coefficient as a function of $\frac{v_{i}}{u_{o}}$

field and at a certain distance from the wing. In order to obtain some estimation let us assume a wing with a certain thickness and covered by a film of ferrofluid in which there are a train of magnets attached below. The minimum skin thickness of a wing depends of several factors, for example, the load distribution, etc, and we can find commercial airplane types, the 727, with minimum skin thickness as low as $0.1 \mathrm{~mm}$ or as high as $1.27 \mathrm{~mm}$ for a DC-8 and DC-9 airplanes. Therefore to be in the safe and most conservative side, let us assume a thickness around $1.27 \mathrm{~mm}$. On the other hand let us consider a ferrofluid with a thickness, say, $2 \mathrm{~mm}$. Therefore, the magnets will be at a distance around $1.27 \mathrm{~mm}+2 \mathrm{~mm}=3.27 \mathrm{~mm}$. Then, we want to measure the magnetic field from a flat arrangement of magnets with a certain total thickness $l$ at a distance $3.27 \mathrm{~mm}$ away from its surface. Finally, it is desired to cover a surface area of the wing 


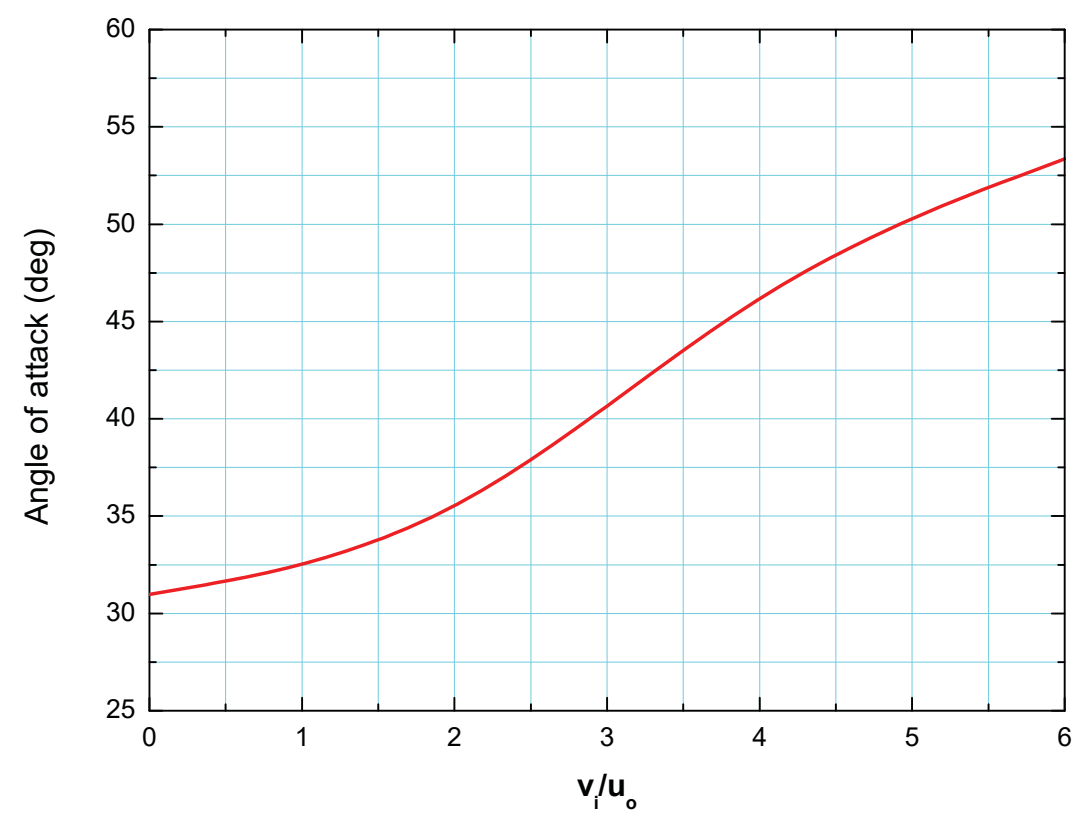

Figure 11. Angle of Attack as a function of $\frac{v_{i}}{u_{o}}$

$S_{w}$ with a ferrofluid film, then the volume of magnet $V_{m}$ needed will be $V_{m}=S_{w} l$, and if the magnet has a density $\rho_{m}$ will result into a weight $W_{m}=\rho_{m} S_{w} l$ and the weight of magnets per unit of surface area of the wing as $\frac{W_{m}}{S_{w}}=\rho_{m} l$. The magnetic field as function of its thickness of the Neodymium magnets, $\rho_{m}=7612 \mathrm{~kg} / \mathrm{m}^{3}$ was measured and the resulting curve is shown in Fig. 13. It is seem that in order to generate the magnetic field around $32 \mathrm{kA} / \mathrm{m}$, or thereabouts, it will require a density around $\frac{W_{m}}{S_{w}}=2.5 \mathrm{~kg} / \mathrm{m}^{2}$. The penalty caused by the excess of weight is small compared with the lift enhancement, and it becomes negligible inasmuch that the total weight of the airplane increase. To see this, let us consider, for example, a DC-8 airplane with a typical weight around $130000 \mathrm{~kg}$. With a lift enhancement around 1.5 this will translates into an additional force around 65000 kilograms-force, however, with a wingspan around 44 meters and a width around 1 meter, the total surface area of the wing will be around 100 $\mathrm{m}^{2}$, or thereabouts, which with the aforementioned calculated figure $\frac{W_{m}}{S_{w}}=2.5 \mathrm{~kg} / \mathrm{m}^{2}$, will result in an extra weight of $2500 \mathrm{~kg}$, i.e., less than a $0.5 \%$. Even assuming the most pessimist figure, the lift gain will be much more higher. It also must be noted that the above roughly calculations is assuming covering the entire wings with a ferrofluid layer, this of course, will be not the case, in fact the ferrofluid film concept will be applied in specific regions for example at the point where the detachment of the boundary layer occurs in order to delay it.

\section{- Declaration of Interests}

The authors report no conflict of interest.

\section{Acknowledgements}

The author thanks Mrs Greenspan D.E as laboratory assistant at the laboratory of department of fluid mechanics at the Polytechnic University of Catalonia (UPC) for the collaboration in the set up of the experiment, measurement and data treatment. This research was supported by the Spanish Ministry of Economy and Competitiveness under 


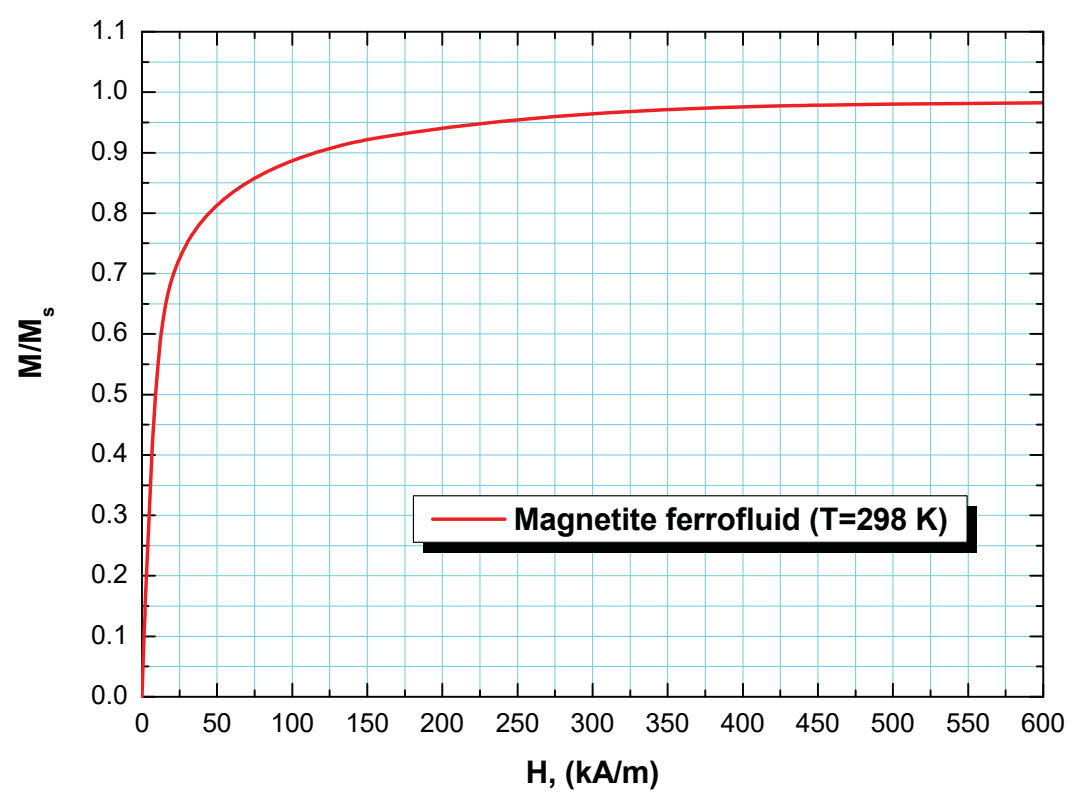

FIGURE 12. Variation of magnetization with the external applied magnetic field

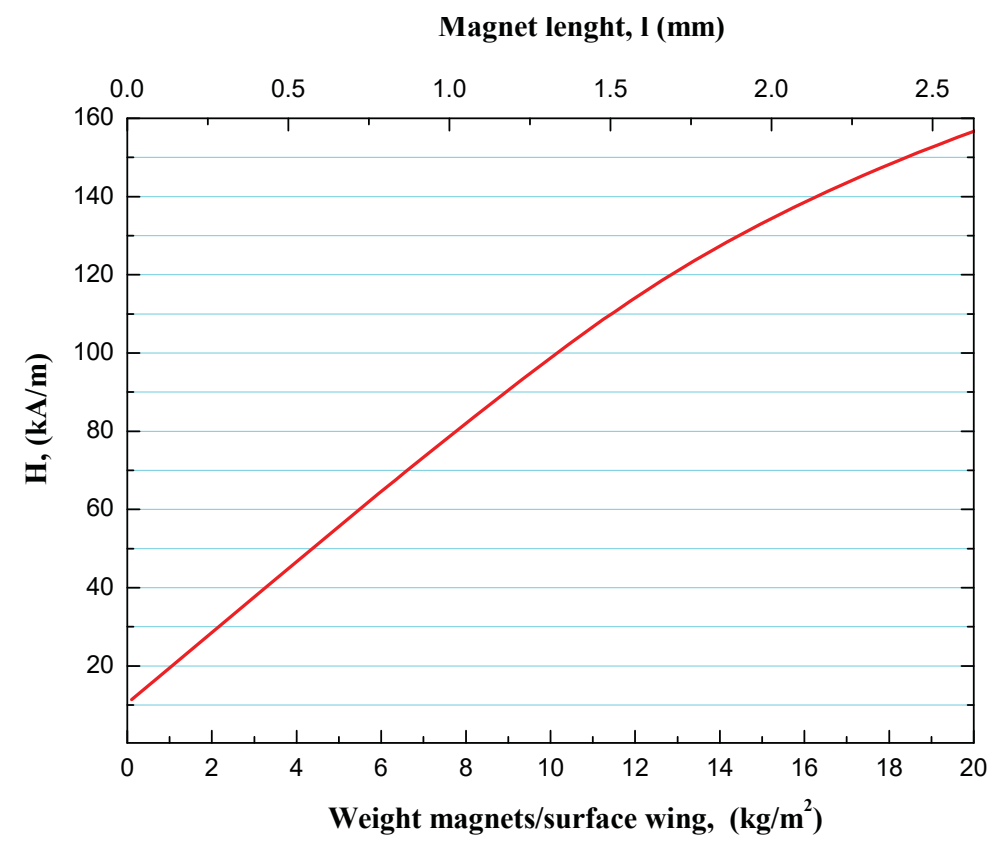

FiguRE 13. Magnetic field generated by a flat magnet at a distance $3.2 \mathrm{~mm}$ form its surface and as function of the thickness of the magnet (upper abscissa) and the weigh of the magnet per unit of surface area of the wing (bottom abscissa).

fellowship grant Ramon y Cajal: RYC-2013-13459.

\section{REFERENCES}

Modi V.J. 1997. Moving surface boundary-layer control : a review. Journal of Fluids and Structures. 11 , p.p. 627-663

Hammer P; Visbal M; Naguib A; Koochesfahani M. 2018. Lift on a steady 2-D symmetric airfoil in viscous uniform shear flow. Journal of Fluid Mechanics, 837, R2.

Fernandez-Feria, R; Alaminos-Quesada, J. 2018. Unsteady thrust, lift and moment of a 
two-dimensional flapping thin airfoil in the presence of leading-edge vortices: A first approximation from linear potential theory. Journal of Fluid Mechanics, 851, 344-373.

Panda J; Zaman K. 1994. Experimental investigation of the flow field of an oscillating airfoil and estimation of lift from wake surveys. Journal of Fluid Mechanics, 265, 65-95. doi:10.1017/S0022112094000765

Bhat S; Zhao J; Sheridan J; Hourigan K; Thompson M. 2019. Evolutionary shape optimisation enhances the lift coefficient of rotating wing geometries. Journal of Fluid Mechanics, 868, 369-384.

Yeh C; Taira K. 2019. Resolvent-analysis-based design of airfoil separation control. Journal of Fluid Mechanics, 867, 572-610.

Gao W; Zhang W; Cheng W; Samtaney R. 2019. Wall-modelled large-eddy simulation of turbulent flow past airfoils. Journal of Fluid Mechanics, 873, 174-210.

Wang S; He G; Liu T. 2019. Estimating lift from wake velocity data in flapping flight. Journal of Fluid Mechanics, 868, 501-537.

Ringuette, M. J., Milano, M., and Gharib, M., 2007. Role of the tip vortex in the force generation of low-aspect-ratio normal flat plates. Journal of Fluid Mechanics, 581, pp. 453-468.

Gilarranz J, Traub L, Rediniotis O., 2005, A new class of synthetic jet actuators-part II: application to flow separation control. Transactions of the ASME-I-Journal of Fluids Engineering, 127(2):377

Rosenweig R.E. 1985. Ferrohydrodynamics. Dover publications, Inc, Mineola, New York.

Landau L.D; Lifshitz. E.M. Fluid Mechanics. Second Edition. 1989. Second edition. Pergamon Press.

Rowell H.S. 1928. Finlayson U.D. Engineering 17, 606 (1922), 23, 249.

Bateman H. 1932. Partial differential equations of mathematical physics. Partial Differential Equations of Mathematical Physics, by H. Bateman, Cambridge, UK: Cambridge University Press, 1932. pp. 175

Prandtl, L. 1938. Zur berechnung der grenzschichten. ZAMM-ournal of Applied Mathematics and Mechanics/Zeitschrift fur Angewandte Mathematik und Mechanik 18.1. 77-82.

Boukenkoul M; Feng-Chen, Li; Wen-Li, Chen; Hong-Na, Zhang. 2017. Lift-generation and moving-wall flow control over a Low Aspect Ratio airfoil. Journal of Fluids Engineering. 140. 